\title{
Subungual onychomycosis due to Aspergillus niger mimicking a glomus tumor: A case report
}

\author{
YUMI MATSUYAMA, TOMOKI NAKAMURA, TOMOHITO HAGI, KUNIHIRO ASANUMA and AKIHIRO SUDO \\ Department of Orthopaedic Surgery, Mie University Graduate School of Medicine, Edobashi, Tsu-city, Mie 514-8507, Japan
}

Received August 3, 2017; Accepted September 8, 2017

DOI: $10.3892 /$ br.2017.994

\begin{abstract}
Onychomycosis is a common nail infection caused by dermatophytes, while non-dermatophytes including Aspergillus spp. are causes of nail onychomycosis. Aspergillus niger is not common as a cause of nail onychomycosis. In the current study we present a 60 -year-old woman with subungual onychomycosis due to Aspergillus niger mimicking a glomus tumor. Physical examination revealed right thumb had a black color of nail bed. Localized tenderness and severe pain were observed. However, the cold sensitivity test, Love's pin test and Hildreth's test were negative. On radiograph, bone erosion was found in a part of distal phalanx at the right thumb. Magnetic resonance imaging identified a mass at the subungual space, which exhibited low signal intensity on T1-weighted images and high signal intensity on T2-weighted images. The differential diagnosis included glomus tumor and infection. The histological findings demonstrated dichotomous septate hyphae. The culture was positive for Aspergillus niger. The results suggested that when physical examination is not typical for a glomus tumor, other diseases may be considered. Additionally, frozen section diagnosis may be useful.
\end{abstract}

\section{Introduction}

Onychomycosis is a common nail infection caused by dermatophytes, while non-dermatophytes including Aspergillus spp. are causes of nail onychomycosis (1-3). Onychomycosis cases caused by Aspergillus spp. are reported to be 2.6-6.1\% (2). However, Aspergillus niger is not common as a cause of nail onychomycosis. There are few reports on onychomycosis caused by Aspergillus niger (2-5). On the other hand, the most common site of glomus tumor is distal phalanx of the fingers, especially in the subungual region $(6,7)$. In the current study, we present the case of a 60-year-old woman diagnosed

Correspondence to: Dr Tomoki Nakamura, Department of Orthopaedic Surgery, Mie University Graduate School of Medicine, 2-174 Edobashi, Tsu-city, Mie 514-8507, Japan

E-mail: tomoki66@clin.medic.mie-u.ac.jp

Key words: onychomycosis, glomus tumor, Aspergillus niger, MRI, subungual with onychomycosis caused by Aspergillus niger, which was initially considered as a glomus tumor on the findings of radiograph and magnetic resonance imaging (MRI).

\section{Case report}

Institutional review board was waved because of completely retrospective case presentation. Informed consent was obtained from the patients for presentation and publication under the protection of personal information.

In March 2014, a 60-year-old woman with diabetes mellitus presented to Mie University Hospital (Tsu-city, Japan) with a 1-month history of pain on her right thumb. She had no history of trauma.

Physical examination revealed the right thumb had a black color of nail bed (Fig. 1). Localized tenderness and severe pain were observed. However, results of the cold sensitivity test, Love's pin test and Hildreth's test were negative. The laboratory studies, revealed that hemoglobin A1c was 7.2\% (standard level; 4.6-7.2\%). C-reactive protein level was within the standard level. Following the radiograph, bone erosion was found in a part of distal phalanx (Fig. 2). MRI identified a mass at subungual space, which exhibited low signal intensity on T1-weighted images and high signal intensity on T2-weighted images (Fig. 3). Gadolinium enhanced MRI was not performed because of allergy. The differential diagnosis included glomus tumor and infection. Excisional biopsy was subsequently performed. Culture and biopsy were performed, because infection was suspected by intraoperative frozen section diagnosis. The histological findings demonstrated dichotomous septate hyphae. Culture was positive for Aspergillus niger. The patient was treated orally with $100 \mathrm{mg}$ itraconazole (Itrizole ${ }^{\circledR}$ ) daily for 3 months. At 6 months, the pigmentation had completely disappeared. On radiograph, bone erosion was healed (Fig. 2).

\section{Discussion}

In this study, we have presented a case with onychomycosis caused by Aspergillus niger, which was initially considered as a glomus tumor. Glomus tumors account for $1-5 \%$ of soft tissue tumors of the hand and $75 \%$ of them are subungual in location (6). The typical clinical triad of localized tenderness, severe pain and cold sensitivity is highly suggestive of glomus tumor (8). In addition to the classical presentation, there are useful tests that help in diagnosing glomus tumor. Cold 


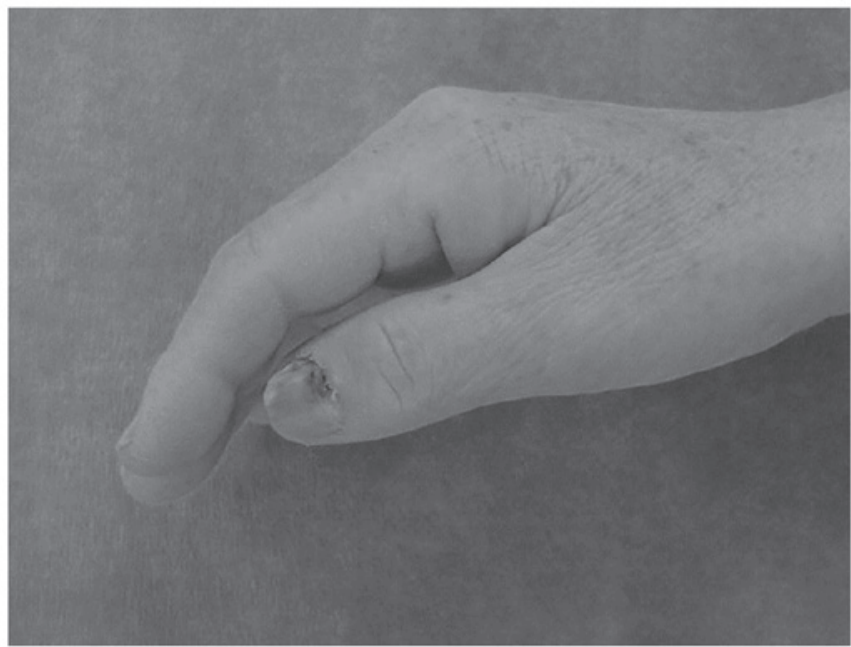

Figure 1. Physical findings. Right thumb had a black color of nail bed.

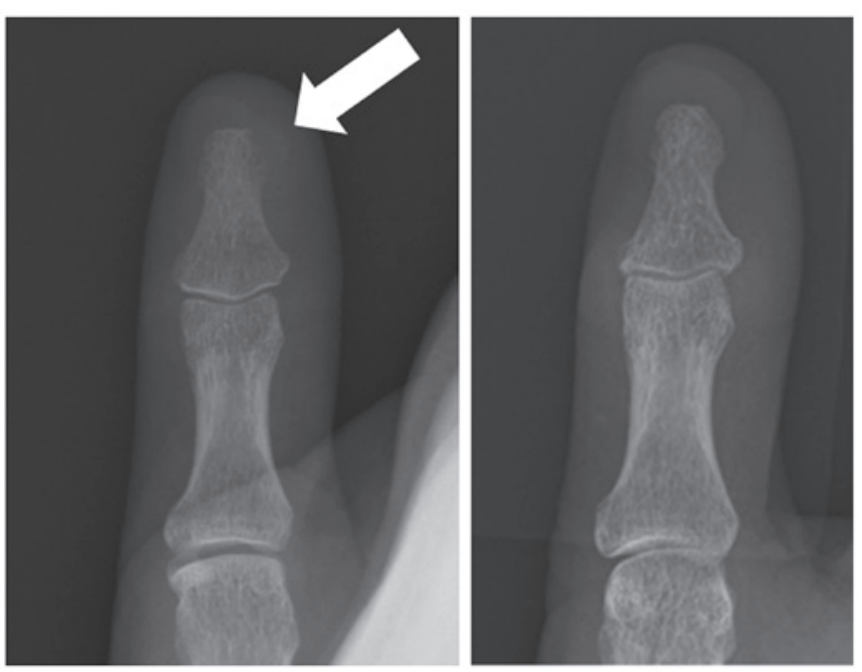

Figure 2. Radiographs of right thumb. (left, preoperative, right, postoperative). sensitivity test was reported to be a reliable test. Bhaskaranand and Navadgi (7) reported sensitivity and specificity of $100 \%$ for glomus tumor although only 14 patients were assessed (7). The Love's pin test and Hildreth's test were also reported to be useful tests for the diagnosis of glomus tumor (6). In the present case, these tests were negative, although localized tenderness and severe pain were found. Furthermore, the mass of the present case was presented as a black nodule. Glomus tumors are usually presented as bluish or pinkish red. It may be necessary to consider other diseases if the findings of these clinical presentations did not coincide with those of glomus tumor.

In some of the cases, cortical thinning or erosive changes are shown in the adjacent bone in radiograph (9). MRI is helpful in making differential diagnosis. It shows low signal intensity on T1-weighted images, high signal intensity on T2-weighted fat-suppression images and enhancement on gadolinium-enhanced images (9). In the present case, bone erosion was shown in the radiograph and MRI showed low signal intensity on T1-weighted images and high signal intensity on T2-weighted images, although gadolinium-enhanced images were not performed. These findings were similar to those of glomus tumor. We consider that the findings of radiographs and MRI result from inflammation induced by onychomycosis at subungual.

Although imaging finding was considered as a glomus tumor, we also consider a possibility of infection as a differential diagnosis because the present case had diabetes mellitus and no response to classical tests such as the cold sensitivity test. Intraoperative frozen section diagnosis led us to perform culture and pathological examination.

Onychomycosis is usually caused by dermatophytes, but some species of non-dermatophytic molds and yeasts are also associated with it. Aspergillus niger is a non-dermatophytic mold that exists as an opportunistic filamentous fungus in all environments. With regard to onychomycosis caused by Aspergillus niger, five cases have been reported. The five cases

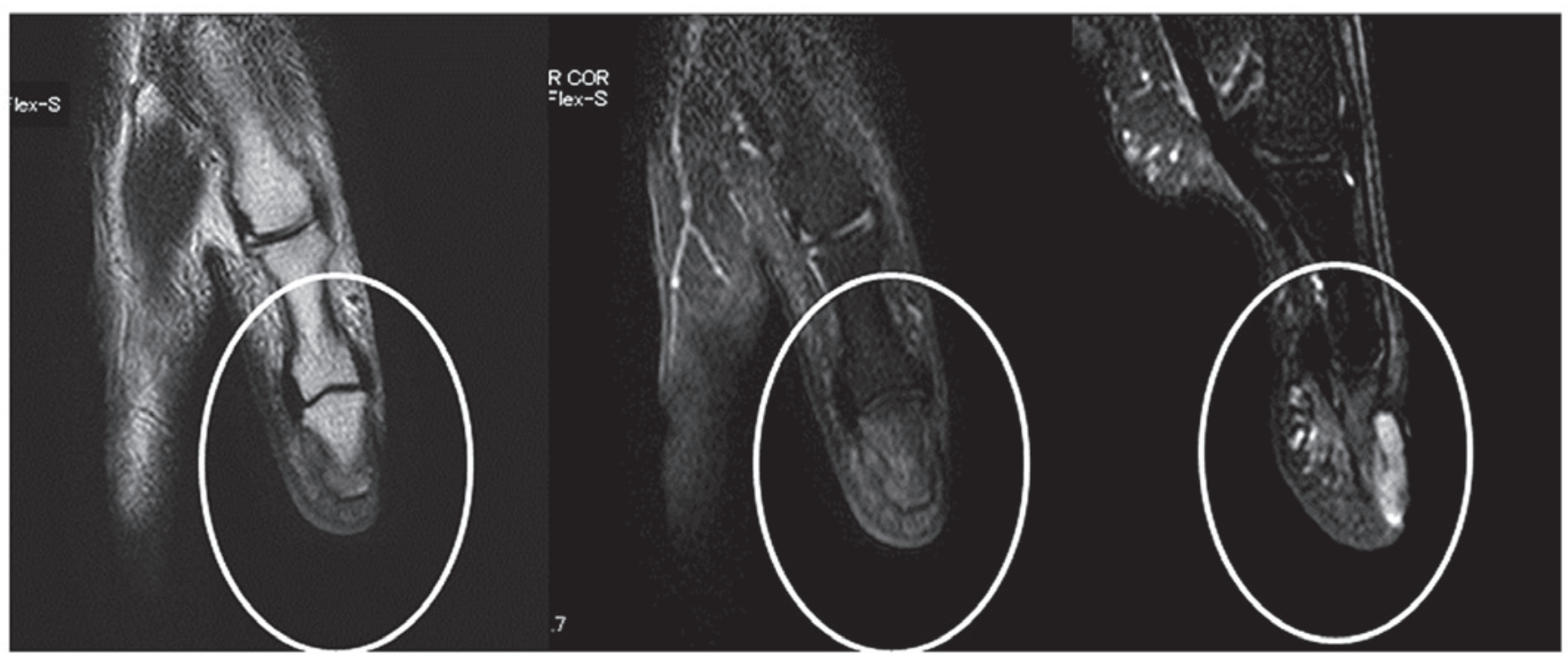

Figure 3. MRI finding at right thumb. (left; T1-weighted images on coronal view, middle; T2-weighted fat-suppression images on coronal view, right; T2-weighted fat-suppression images on sagittal view). MRI, magnetic resonance imaging. 
occurred in adults aged 39-66 years (2-5). Four cases involved toe nails and one case involved fingernails. Onychomycosis caused by Aspergillus niger shows black pigment. This discoloration was caused by the black candida of Aspergillus niger in nail keratin, which clinically suggest a diagnosis of onychomycosis, although one case did not show black pigment (4).

In conclusion, we present a patient with onychomycosis mimicking a glomus tumor. When classical presentations are not found for a glomus tumor, other diseases may be considered. Intraoperative frozen section diagnosis may be useful.

\section{References}

1. Nouripour-Sisakht S, Mirhendi H, Shidfar MR, Ahmadi B, Rezaei-Matehkolaei A, Geramishoar M,Zarei F and Jalalizand N: Aspergillus species as emerging causative agents of onychomycosis. J Mycol Med 25: 101-107, 2015.
2. Kim DM, Suh MK, Ha GY and Sohng SH: Fingernail onychomycosis due to Aspergillus niger. Ann Dermatol 24: 459-463, 2012.

3. Noguchi H, Hiruma M, Miyashita A, Makino K, Miyata K and Ihn H: Ihn Hironobu. A case of fingernail onychomycosis due to Aspergillus flavus. Med Mycol J 57: E21-E25, 2016.

4. Tamer F and Yuksel ME: Onychomycosis due to Aspergillus niger without black nail discoloration: A case report. Our Dermatol Online 8: 233-234, 2017.

5. Tosti A and Piraccini BM: Proximal subungual onychomycosis due to Aspergillus niger: Report of two cases. Br J Dermatol 139: 156-157, 1998.

6. Morey VM, Garg B and Kotwal PP: Glomus tumours of the hand: Review of literature. J Clin Orthop Trauma 7: 286-291, 2016.

7. Bhaskaranand $\mathrm{K}$ and Navadgi BC: Glomus tumour of the hand. J Hand Surg Br 27: 229-231, 2002.

8. Carroll RE and Berman AT: Glomus tumors of the hand: Review of the literature and report on twenty-eight cases. J Bone Joint Surg Am 54: 691-703, 1972.

9. Baek HJ, Lee SJ, Cho KH, Choo HJ, Lee SM, Lee YH, Suh KJ, Moon TY, Cha JG, Yi JH, et al: Subungual tumors: Clinicopathologic correlation with US and MR imaging findings. Radiographics 30: 1621-1636, 2010. 